5 Masauzi N, Ichikawa S, Nishimura F, et al. Primary angiosarcoma of the right atrium detected by magnetic resonance imaging. Intern Med 1992; 31: 1291-1297.

6 Ito K, Kubota K, Morooka M, et al. Diagnostic usefulness of 18FFDG PET/CT in the differentiation of pulmonary artery sarcoma and pulmonary embolism. Ann Nucl Med 2009; 23: 671-676.

7 Chung JH, Cho KJ, Lee SS, et al. Overexpression of Glut1 in lymphoid follicles correlates with false-positive (18)F-FDG PET results in lung cancer staging. J Nucl Med 2004; 45: 999-1003.

8 Medford AR, Bennett JA, Free CM, et al. Endobronchial ultrasound guided transbronchial needle aspiration. Postgrad Med J 2010; 86: 106-115.
9 Aumiller J, Herth FJ, Krasnik M, et al. Endobronchial ultrasound for detecting central pulmonary emboli: a pilot study. Respiration 2009; 77: 298-302.

10 Kim JB, Kim SH, Lim SY, et al. Primary angiosarcoma of the pulmonary trunk mimicking pulmonary thromboembolism. Echocardiography 2010; 27: E23-E26.

11 Wallace MB, Woodward TA, Raimondo M, et al. Transaortic fineneedle aspiration of centrally located lung cancer under endoscopic ultrasound guidance: the final frontier. Ann Thorac Surg 2007; 84: 1019-1021.

\title{
Ralstonia mannitolilytica and COPD: a case report
}

\section{To the Editors:}

Ralstonia mannitolilytica is a recently established species of clinical significance and was previously known as Pseudomonas thomasii or Ralstonia pickettii biovar 3/thomasii [1]. It has been recovered from the respiratory tract of patients with cystic fibrosis and has also been associated with catheter-associated bacteraemia, recurrent meningitis, infection of a haemoperitoneum, urinary tract infection and post-renal transplant infection. Hospital outbreaks of $R$. mannitolilytica due to contamination of water [2], saline solutions [3] or oxygen-delivery devices [4] have also been reported. However, this bacterium has not been reported in patients with respiratory illnesses other than cystic fibrosis.

Isolate G100 was recovered from a sputum sample from a male, 78-yr-old patient in April, 2010. This patient was presented with cough and gradually worsening dyspnoea for 1 month, but without fever. He received no antimicrobial agents prior to admission. This patient had a 20-yr history of intermittent cough, and chronic obstructive pulmonary disease (COPD) was diagnosed 10 yrs previously. He had also had type II diabetes mellitus for $5 \mathrm{yrs}$ and had been a cigarette smoker for $>20 \mathrm{yrs}$, but had stopped smoking 10 yrs previously. Physical examination revealed a "barrel-shaped" chest, reduced breath sounds and crackles. On admission, a full blood count revealed haemoglobin $15.1 \mathrm{~g} \cdot \mathrm{dL}^{-1}$, white cell count $5.93 \times 10^{9}$ cells $\cdot \mathrm{L}^{-1}$ (neutrophils $4.49 \times 10^{9}$ cells $\cdot \mathrm{L}^{-1}$ and lymphocytes $\left.1.25 \times 10^{9} \mathrm{cells} \cdot \mathrm{L}^{-1}\right)$ and platelets $187 \times 10^{9}$ cells $\cdot \mathrm{L}^{-1}$. Routine serum chemistry was normal. Blood-gas analysis revealed $\mathrm{pH} 7.28$, oxygen tension $64 \mathrm{mmHg}$, carbon dioxide tension $80 \mathrm{mmHg}, \mathrm{HCO}_{3}^{-} 37.6 \mathrm{mmol} \cdot \mathrm{L}^{-1}$ and arterial oxygen saturation $89 \%$, suggesting type II respiratory failure and respiratory acidosis. High-resolution chest computed tomography on admission revealed barrel-shaped chest and increased lung markings, but no infiltrations. He was diagnosed with an acute exacerbation of COPD and a sputum sample was sent on admission, from which G100 was isolated. The sputum sample was of good quality when examined by microscopy and Gram-negative rods were detected.

G100 was identified as a Ralstonia sp. of Centers for Disease Control group II using a MicroScan Walkaway 96 SI automated system (Siemens Healthcare Diagnostics, Deerfield, IL, USA). Species identification was performed by partially sequencing the $16 \mathrm{~S}$ ribosomal RNA (rRNA) gene amplified with universal primers $27 \mathrm{~F}$ and $1492 \mathrm{R}$ [5]. The 1,405-bp partial 16S rRNA sequence of G100 was identical to that of $R$. mannitolilytica strain AU0428 from a cystic fibrosis patient in the USA (GenBank accession number AY043378) [6], strains LMG 19090 (LBV407; AJ270257) and LMG 19091 (LBV371; AJ270256) from cases of recurrent meningitis and haemoperitoneum infection in Belgium [1], and many uncultured clones (e.g. GQ417788) from environmental samples in France.

Random amplification of polymorphic DNA (RAPD) typing has been used previously to determine the clonal relatedness of $R$. mannitolilytica isolates. Using the R. pickettii RAPD primers P3 (5'-AGACGTCCAC-3') and P15 (5'-AATGGCGCAG-3') [7], 30 R. mannitolilytica clinical isolates from Austria have been classified four distinct genotypes [8]. RAPD using P3 and P15 was performed as described previously [7], revealing that G100 belonged to a genotype different from those seen in Austria (data not shown).

G100 was resistant to amikacin, gentamicin, tobramycin, ampicillin, ampicillin/sulbactam, amoxicillin/clavulanate, piperacillin, ticarcillin/clavulanate, cefazolin, ceftazidime, cefoxitin and aztreonam, intermediate to cefotaxime and cefepime, and sensitive to ceftriaxone, piperacillin/tazobactam, imipenem, ciprofloxacin, levofloxacin and trimethoprim/sulphamethoxazole, as determined with the Walkaway system using Negative Combo panel type 31.

Piperacillin/tazobactam (Wyeth, Beijing, China), $4.5 \mathrm{~g}$ every $8 \mathrm{~h}$, was initiated as empirical therapy and continued after the culture result was available, since G100 was sensitive to this compound. This patient also received noninvasive ventilation. However, The patient's condition gradually deteriorated. Therefore, after 9 days of therapy, piperacillin/tazobactam was replaced by panipenem/betamipron (Daiichi Sankyo, Beijing, China) but patient's condition did not improve. After 1 month of hospitalisation, the patient received endotracheal intubation and was transferred to the intensive care unit (ICU) due to deteriorating respiratory failure. Various microorganisms, including Aspergillus spp., Candida albicans, Klebsiella oxytoca, Klebsiella planticola, Acinetobacter baumannii and Pseudomonas aeruginosa, were recovered from the sputum samples collected at different time-points before or after invasive ventilation. 
Although antibacterial (e.g. cefepime, cefoperazone/sulbactam and moxifloxacin) and antifungal agents (itraconazole) were administered intravenously according to the microbiological results, the patient had no response and finally died of respiratory failure after a 28-day ICU stay.

The isolation of G100 was unlikely to have been due to contamination, based on the following observations. First, the isolation of Ralstonia spp. from clinical samples is extremely rare at our centre and no other cultures in West China Hospital grew Ralstonia spp. in this year. Secondly, no saline solutions or water were used to collect the sputum sample, which was collected prior to noninvasive ventilation. Thirdly, Gram-negative rods were detected in the sputum by microscopy. As G100 was recovered from a sample collected on admission and this patient had no recent history of hospitalisation, this isolate probably had a community origin. After administration of piperacillin/tazobactam, no $R$. mannitolilytica was isolated from sputum samples collected from this patient afterwards, but the patient's condition did not improve. This suggests that G100 was probably not the causative agent, or at least, not the sole causative agent of the acute exacerbation of COPD in this patient. It is more likely that $R$. mannitolilytica had colonised in the respiratory tract in this case.

R. mannitolilytica has been recovered from the respiratory tract of patients with cystic fibrosis [6]. However, cystic fibrosis is not common in China and this patient did not have this disease. As microbial colonisation of airways could lead to exacerbations of COPD, the isolation of $R$. mannitolilytica in the respiratory tract of the COPD patient could be of clinical importance. As misidentification is common, $R$. mannitolilytica might be an overlooked member of the bacterial flora in the respiratory tract of COPD patients. Ralstonia spp. (identified as R. pickettii, but without describing the method for identification) have previously been found to cause COPD exacerbation [9]. Nonetheless, our report describes a rare case of $R$. mannitolilytica associated with a COPD patient and, to our knowledge, this case is the first identification of $R$. mannitolilytica from clinical samples in mainland China.

\section{Z.Y. Zong* and C.H. Peng",}

*Center of Infectious Diseases, "Dept of Respiratory Medicine, West China Hospital, Sichuan University, Chengdu, and “Dept of Emergency Medicine, People's Hospital of Guizhou Province, Guiyang, China.

Correspondence: Z.Y. Zong, Center of Infectious Diseases, West China Hospital (Huaxi), Guoxuexiang 37, Chengdu 610041, China. E-mail: zongzhiy@scu.edu.cn

Statement of Interest: None declared.

\section{REFERENCES}

1 De Baere T, Steyaert S, Wauters G, et al. Classification of Ralstonia pickettii biovar 3/'thomasii' strains (Pickett 1994) and of new isolates related to nosocomial recurrent meningitis as Ralstonia mannitolytica sp. nov. Int J Syst Evol Microbiol 2001; 51: 547-558.

2 Baird RM, Elhag KM, Shaw EJ. Pseudomonas thomasii in a hospital distilled-water supply. J Med Microbiol 1976; 9: 493-495.

3 Pan HJ, Teng LJ, Tzeng MS, et al. Identification and typing of Pseudomonas pickettii during an episode of nosocomial outbreak. Zhonghua Min Guo Wei Sheng Wu Ji Mian Yi Xue Za Zhi 1992; 25: 115-123.

4 Jhung MA, Sunenshine RH, Noble-Wang J, et al. A national outbreak of Ralstonia mannitolilytica associated with use of a contaminated oxygen-delivery device among pediatric patients. Pediatrics 2007; 119: 1061-1068.

5 Lane DJ. 16S/23S rRNA sequencing. In: Stackebrant E, Goodfellow $\mathrm{M}$, eds. Nucleic acid techniques in bacterial systematics. New York, John Wiley \& Sons, 1991; pp. 115-175.

6 Coenye T, Vandamme P, LiPuma JJ. Infection by Ralstonia species in cystic fibrosis patients: identification of $R$. pickettii and $R$. mannitolilytica by polymerase chain reaction. Emerg Infect Dis 2002; 8: 692-696.

7 Maroye P, Doermann HP, Rogues AM, et al. Investigation of an outbreak of Ralstonia pickettii in a paediatric hospital by RAPD. J Hosp Infect 2000; 44: 267-272.

8 Daxboeck F, Stadler M, Assadian O, et al. Characterization of clinically isolated Ralstonia mannitolilytica strains using random amplification of polymorphic DNA (RAPD) typing and antimicrobial sensitivity, and comparison of the classification efficacy of phenotypic and genotypic assays. J Med Microbiol 2005; 54: 55-61.

9 Sancho-Chust JN, Andreu AL, Chiner E. Ralstonia picketti in chronic obstructive pulmonary disease exacerbation. Arch Bronconeumol 2010; 46: 47-48.

\section{Can transmissible strains of Pseudomonas aeruginosa be successfully eradicated?}

\section{To the Editors:}

Recent microbiological surveillance using molecular typing (genotyping) has provided compelling evidence for Pseudomonas aeruginosa cross-infection at many European, Australian and Canadian cystic fibrosis (CF) centres [1-4]. The transmissible strains responsible for this cross-infection pose an increased risk for acquisition of infection for patients currently free of $P$. aeruginosa. As transmissible strains are often resistant to multiple antibiotics, they may also be more difficult to eradicate. At present, there is a paucity of evidence regarding this. We therefore report the efficacy of eradication therapy in a cohort of six patients who acquired a transmissible strain as their first isolate of $P$. aeruginosa. 